\title{
PHILIPPE SÉNAC
}

\section{Quelques remarques sur l'historiographie récente de la frontière dans l'Espagne médiévale ( $\mathrm{VIII}^{\mathrm{e}}-\mathrm{XIII}{ }^{\mathrm{e}}$ siècle)}

Sans doute avivée par les nombreux bouleversements territoriaux qui firent suite à la guerre froide, à l'effondrement du mur de Berlin ou à l'éclatement de l'ancienne Yougoslavie, la question de la frontière a connu ces dernières décennies un très vif regain d'intérêt en Europe. Célébré par de nombreux colloques, ce thème de recherche a en effet été abordé non seulement par des historiens, mais également par des géographes et des sociologues dans le cadre d'une réflexion plus globale concernant les limites et le phénomène des discontinuités ${ }^{1}$. Nulle part cependant la question de la frontière n'a connu autant de succès qu'en Espagne, en particulier après 1976 et la loi sur les autonomies. Pour la période médiévale, un essai de synthèse réalisé en 1995 avec André Bazzana et Pierre Guichard lors d'un colloque de la série »Castrum« nous avait conduit à comptabiliser plus d'un millier d'ouvrages et d'articles relatifs à la frontière dans la péninsule Ibérique ${ }^{2}$, et ce volume s'est encore accru, en particulier à la suite de la publication de plusieurs thèses comme celle d'Eduardo Manzano Moreno, »La frontera de al-Andalus en época de los omeyas« (1991), de Philippe Sénac, »La frontière et les hommes du VIII ${ }^{\mathrm{e}}$ au XII ${ }^{\mathrm{e}}$ siècle« (1997), celle de Pascal Buresi, »La frontière entre chrétienté et islam dans la péninsule Ibérique du Tage à la sierra Morena de la fin du XI ${ }^{\mathrm{e}}$ au milieu du XIII siècle« (2000) ou encore, pour des périodes plus anciennes, celle de Céline Martin sur »La géographie du pouvoir dans l'Espagne wisigothique« $(2000)^{3}$.

1 Voir en particulier Laurent Carroue, Paul Claval, Guy Di Meo, Limites et discontinuités en géographie, Paris 2002. Il n'est peut-être pas inutile de rappeler la définition du mot »frontière « que donnait Michel Foucher, L'invention des frontières, Paris 1986 (Les sept épées, 41), p. 59: »Les frontières sont d'abord l'enveloppe continue d'un ensemble spatial, d'un État, qui a atteint suffisamment de cohésion politique interne et d'homogénéité économique pour que les clivages principaux ne traversent plus l'intérieur du territoire et la collectivité humaine, mais aient été rapportés, par changement d'échelle, en position limite... Les frontières sont dans le même temps un plan de séparation-contact ou, mieux, de différenciation des rapports de contiguïté avec d'autres systèmes politiques, qui ne sont pas forcément de même nature ni du même degré d'élaboration«.

2 André BaZZAna, Pierre Guichard, Philippe SÉnAC, La Frontière dans l'Espagne médiévale, dans: Jean-Michel POISSON (dir.), Castrum IV. Frontière et peuplement dans le monde méditerranéen au Moyen Âge, Rome, Madrid 1992 (Collection de la Casa de Velázquez, 38), p. 35-59.

3 Eduardo ManZANo Moreno, La frontera de al-Andalus en época de los omeyas, Madrid 1991 (Biblioteca de historia, 9); Philippe SÉNAC, La frontière et les hommes (VIII $-\mathrm{XII}^{\mathrm{e}}$ siècle). Le peuplement musulman au nord de l'Èbre et les débuts de la reconquête aragonaise, Paris 2000; Pascal BURESI, La frontière entre chrétienté et islam dans la péninsule Ibérique du Tage à la sierra Morena (fin $\mathrm{XI}^{\mathrm{e}}-$ milieu XIII ${ }^{\mathrm{e}}$ siècle), Paris 2004; Céline MARTIN, La géographie du pou- 
Cet engouement croissant pour la notion de »frontière« ne s'explique pas seulement par des raisons circonstancielles ou politiques, comme la question basque ou le particularisme catalan. Il s'explique principalement par le fait que toute la péninsule Ibérique fut une terre de frontière et que cette notion résume parfaitement l'histoire médiévale de l'Espagne comme l'avait déjà suggéré un livre d'Angus Mackay intitulé »Spain in the Middles Ages« et sous-titré »From Frontier to Empire « ${ }^{4}$. De fait, depuis la conquête arabo-berbère du début du VIII ${ }^{\mathrm{e}}$ siècle jusqu'à la chute de l'émirat nasride de Grenade en 1492, toute l'histoire de la péninsule Ibérique fut celle d'un front mouvant opposant deux forces, voire deux civilisations, l'islam et la chrétienté. Cette spécificité hispanique s'est affirmée à l'époque du franquisme, à la suite d'un livre publié en 1954 sous le titre »El concepto de España en la Edad Media«, dans lequel José Antonio Maravall affirmait qu'aucune histoire médiévale en Europe n'était semblable à celle de l'Espagne. Il avançait ainsi que »probablement il n'existe aucun pays où s'est produit un fait aussi extraordinaire que celui qu'on appelle généralement une grande partie du Moyen Âge« et, un peu plus loin, »il n'existe rien [...] en Europe qui soit comparable à la Reconquista espagnole « ${ }^{5}$.

Le thème de la frontière continue de susciter beaucoup d'intérêt comme le montre le libellé d'une récente question d'agrégation d'histoire intitulée »Les relations des pays d'islam avec le monde latin (milieu $\mathrm{X}^{\mathrm{e}}$-milieu XIII ${ }^{\mathrm{e}}$ siècle)«, un colloque organisé à Madrid et Tolède en 2001 sur »Identidad y representación de la frontera en la España medieval (siglos XI-XIV)«, ou encore l'ouvrage récent de José Rodríguez Molina, »La vida de moros y cristianos en la Frontera«, publié en $2007^{6}$, et enfin le titre d'un colloque organisé par la fondation Sánchez Albornoz à León à la fin de l'année 2007, „Cristianos y Musulmanes en la península ibérica: la guerra, la frontera y la convivencia«? Les combats et les relations entretenues pendant la >reconquête < continuent de faire l'objet de recherches universitaires, comme celles menées par Miguel Ángel Ladero Quesada, Carlos de Ayala Martínez ou Francisco García Fitz, mais, sous l'effet du

voir dans l'Espagne wisigothique, Lille 2003 (Histoire et civilisations, 861). Voir aussi le volume de Klaus HERBERS, Nikolas JASPERT (dir.), Grenzräume und Grenzüberschreitungen im Vergleich. Der Osten und der Westen des mittelalterlichen Lateineuropa, Berlin 2007 (Europa im Mittelalter, 7).

4 Angus MacKay, Spain in the Middles Ages. From Frontier to Empire, London 1977.

5 José Antonio MARAVALl, El concepto de España en la Edad Media, Madrid ${ }^{3} 1981$ (Colección estudios politicos, 2), p. 249-250: »probablemente no hay otro país en el que se dé un hecho tan extraordinario como el que representa aquel que, llenando todo el periodo de nuestra Edad Media, se ha llamado >la Reconquista««; et »Nada hay [...] que se parezca en Europa a la Reconquista española«. Cette idée reprend celle formulée quelques années plus tôt par Ramon MENÉNDEZ PIDAL, La España del Cid, Madrid 1929, vol. 1, p. 73, qui présente »la idea reconquistadora como definición de nuestra Edad Media«.

6 José Rodríguez Molina, La vida de moros y cristianos en la frontera, Alcalá de Henares 2007 (Colección almayar, 2).

7 Cristianos y musulmanes en la Península ibérica: la guerra, la frontera y la convivencia. XI congreso de estudios medievales, 2007, León 2009. De manière très suggestive, l'histoire des communautés juives pendant la Reconquête vient même d'être abordée au travers du thème de la frontière par Jonathan RAY, The Sephardic Frontier. The Reconquista and the Jewish Community in Medieval Iberia, New York 2006. 
succès des enquêtes archéologiques et des études concernant al-Andalus, il semble que les travaux aient évolué vers des problématiques à la fois plus matérielles et plus sociales.

Il serait à la fois impossible et fastidieux de dresser ici un bilan exhaustif de tous les travaux relatifs à la frontière dans l'Espagne médiévale comme au Portugal, récemment éclairé par les études de Stéphane Boissellier et de Christophe Picard ${ }^{8}$, et l'objectif des pages qui suivent consistera plutôt à tenter une synthèse des recherches les plus récentes tant du côté chrétien que du côté musulman de la frontière pour proposer finalement quelques thèmes de recherche encore peu ou mal explorés. Pour cela, je centrerai mon propos sur les périodes antérieures à l'émergence d'une limite frontalière semblable à celle que connurent les époques moderne et contemporaine, c'est-àdire vers le milieu du XIII ${ }^{\mathrm{e}}$ siècle lorsque Castillans et Nasrides se trouvent face à face, en accordant une attention particulière aux régions que je connais le mieux, c'est-à-dire l'ensemble de la vallée de l'Èbre, des confins de la Castille à la Méditerranée.

\section{LA FRONTIÈRE ET SES NOMS}

Du côté musulman, les controverses qui opposèrent autrefois Joaquin Bosch Vila et Husayn Monés se sont tues et on admet généralement que le terme le plus communément employé par les auteurs arabes pour désigner les périphéries d'al-Andalus était le mot »tagir « (pl. »țugūur«). L'époque omeyyade en connaissait trois: la Marche inférieure, dont le chef-lieu était Mérida, la Marche moyenne, dont le chef-lieu fut transféré en 936 de Tolède à Medinaceli, et enfin la Marche supérieure, qui se développait autour de Saragosse. La date à laquelle cette partition de l'espace apparut est mal définie dans la mesure où le mot »tağr« est généralement utilisé par des auteurs largement postérieurs aux événements qu'ils relatent. Tout porte cependant à considérer que cette appellation surgit sous l'effet d'une résistance ennemie ou d'une pression inverse, c'est-à-dire non pas du temps de la conquête mais vers la fin du VIII ${ }^{\mathrm{e}}$ siècle, en particulier après les offensives franques sur Barcelone, Lérida et Huesca, et après le raid d'Alphonse II des Asturies sur Lisbonne en 798. Il s'agissait alors non pas de frontières linéaires, mais de frontières zonales, la notion de limite dans al-Andalus comme dans le reste du monde arabo-musulman étant inconnue. Sans doute le géographe al-Mas'ūdī précise-t-il au milieu du $X^{\mathrm{e}}$ siècle que $» d e$ nos jours, en 332/943, la marche-frontière des musulmans, à l'Est de l'Espagne, passe à Tortose sur la côte de la Méditerranée, puis du même côté, en tirant vers le Nord, à Fraga, qui est [bâtie] sur une rivière et

8 Stéphane BoisSELLIER, Naissance d'une identité portugaise. La vie rurale entre Tage et Guadiana, de l'islam à la Reconquête ( $\mathrm{X}^{\mathrm{e}}-\mathrm{XIV}^{\mathrm{e}}$ siècles), Paris 1999; ID., Le peuplement médiéval dans le sud du Portugal: constitution et fonctionnement d'un réseau d'habitats et de territoires $\left(\mathrm{XII}^{\mathrm{e}}-\mathrm{XV}^{\mathrm{e}}\right.$ siècles), Paris 2003; Christophe PICARD, Le Portugal musulman (VIII ${ }^{\mathrm{e}}-\mathrm{XIII}{ }^{\mathrm{e}}$ siècle).

L'Occident d'al-Andalus sous domination islamique, Paris 2000. 
enfin à Lérida «9. Mais ceci révèle surtout qu'il existait autant de frontières que de villes fortifiées, un peu comme en Orient avec les 'awāssim dressées face à Byzance. La conquête n'avait pas de limite et ce n'est que longtemps après les premiers revers musulmans que l'on vit surgir des mentions de bornes destinées à expliquer l'échec des combattants: ainsi, selon plusieurs auteurs comme az-Zuhrī, Ibn al-Ațīr et al-Ḥimyarī, Mūsā b. Nușayr aurait trouvé en entrant en Gaule un temple ou une statue avec une inscription en arabe dont le texte disait: »fils d'Ismā̄îl, faites demi-tour. Vous ne pouvez aller plus loin. Je vous en donnerai l'explication si vous me le demandez, mais si vous ne rebroussez pas chemin, vous vous entretuerez jusqu'à la fin des siècles « ${ }^{10}$. Une évolution se fait cependant jour à partir de la seconde moitié du XIII ${ }^{\mathrm{e}}$ siècle, sous l'effet de la pression castillane, lorsque les sources arabes empruntent aux chrétiens le mot »frontière« sous la forme farantīra. Avec l'émergence de l'émirat nasride, on passe alors d'une frontière zonale à une ligne fortifiée, en particulier du temps du sultan Muhammad V (1354-1391). Il n'est cependant pas impossible que l'idée d'une ligne de défense conçue comme un limes soit plus ancienne comme le suggèrent certains passages du Tibyān où l'émir 'Abd Allāh de Grenade cherche à édifier une ligne défensive hérissée de huușün face à Alméria.

Les choses sont plus complexes du côté chrétien. Au point de départ de l'évolution sémantique figure la monarchie wisigothique dans laquelle, selon Céline Martin, les textes latins de ce temps emploient alors les termes de »fines « (au pluriel), de »confinium«, plus rarement celui de »clausura«, en particulier dans les zones de montagnes.

9 Al-Mas'ūdī, murūğ ad-dahab wa ma'ādin al-ğawhar/Les prairies d'or, éd./trad. Charles BARBIER de Meynard, Abel Pavet de Courteille, Charles Pellat, vol. 1, § 403, Beyrouth 1966/Paris 1962, p. 193 (éd. arabe), p. 147 (trad. française).

10 Évariste LÉVI-PROVEnÇAL, La péninsule Ibérique au Moyen Âge d'après le kitāb ar-rawḍ almi'țār fì habar al-aqțār d'Ibn 'Abd al-Mun'im al-Himyari, Leyde 1938, p. 34; az-Zuhrī, kitāb al-ğa'rāfiyya, texte arabe publié par Mohamed HADJ-SADOK, dans: Bulletin d'études orientales XXI (1968), p. 230: »À Narbonne se trouvait une statue sur laquelle était écrit: fils d'Ismā̄îl, faites demi-tour. Vous ne pouvez aller plus loin. Je vous en donnerai l'explication si vous me le demandez, mais si vous ne rebroussez pas chemin, vous vous entretuerez jusqu'à la fin des siècles [trad. PS] «. La version d'Ibn al-Ațīr est très proche: »Moûsa alla conquérir Saragosse et les villes qui en dépendent; puis il pénétra dans le pays des Francs, où il parvint jusqu'à une vaste plaine déserte, mais où se trouvaient des monuments, entre autres une idole debout, sur laquelle étaient gravés ces mots: Fils d'Ismā'īl, c'est ici votre point extrême, et il vous faut retourner. Si vous me demandez à quel lieu vous retournez, je vous répondrai que c'est aux discussions relativement à ce qui vous concerne, si bien que vous vous couperez la tête les uns les autres, ce qui a eu lieu déjà [trad. PS] «. Annales du Maghreb et de l'Espagne, dans: Revue africaine $224 / 1^{\text {er }}$ trimestre (1897), p. 18. Ibn Id̄ārī reprend également cette version: »Moûsa arriva jusqu'à une statue portant écrit sur sa poitrine: fils d'Ismâ'îl, c'est ici votre point extrême. Si vous me demandez où vous retournerez, nous vous apprendrons que c'est à des discussions entre vous, si bien que vous vous couperez le cou les uns aux autres«. Al-Bayano'l-Mogrib (albayān al-mugrib), Histoire de l'Afrique et de l'Espagne traduite et annotée par Edmond FAGNAN, vol. 2, Alger 1904, p. 25. Al-Himyarī évoque aussi cette tradition: »Mūsā ibn Nușayr [...] s'enfonça donc dans le pays d'Ifranğa et finit par arriver dans une vaste région de plaine, remplie de vestiges antiques. Il y trouva un grand temple qui se dressait à la manière d'une colonne et qui portait, gravée en arabe, une inscription, dont le texte, qu'on lut, disait: >vous voici, ô fils d'Ismā'îll, parvenus au terme de votre avance! Retournez-vous en` [trad. PS]«. 
Elle ajoute que la frontière dans la monarchie wisigothique était plus zonale que linéaire ${ }^{11}$, même si les recherches archéologiques récentes, dans le massif des Albères, en Navarre et dans les Asturies suggèrent plutôt l'existence de lignes de forteresses.

\section{LA FRONTIÈRE CATALANE}

Quelques décennies plus tard, avec l'apparition de petites principautés chrétiennes dans le nord de la Péninsule et l'expansion franque au-delà des Pyrénées, les termes employés par les sources latines traduisent une évolution sensible. Ainsi, en Catalogne, les travaux de Michel Zimmermann ont montré que le vocable le plus fréquemment employé était celui de »marca«. Il s'agissait alors tout au long des $\mathrm{IX}^{\mathrm{e}}$ et $\mathrm{X}^{\mathrm{e}}$ siècles d'une frontière zonale (marca hispanica) qui avait elle-même ses limites en fonction de la proximité des musulmans (in extremis finibus marchiarum contra Ispaniam). Il s'agissait d'un espace désert, seulement fréquenté par des renégats, des païens, des aventuriers, des esclaves en fuite et des vagabonds souvent qualifiés de »maligni homines «. Terre de danger, cet espace devint cependant une terre attractive, une zone pionnière où la mise en valeur des terres était synonyme de liberté. La thèse d'Henri Dolset, soutenue à Toulouse en 2004, a révélé que le mot »frontera « n'y était pas complètement inconnu mais qu'il désignait une voie servant de confront, une berge de rivière, ou, aux $\mathrm{XI}^{\mathrm{e}}$ et $\mathrm{XII}^{\mathrm{e}}$ siècles, le rivage de la mer. Le mot ne désignait jamais les terres placées au voisinage des musulmans et, dans un cas seulement, "frontière" figure comme limite de territoire (frontera de sua terra), en 1057, dans une liste de griefs formulée par le comte Raimond Bérenger I ${ }^{\mathrm{er}}$ de Barcelone contre Guillem II de Besalu $^{12}$.

\section{LA FRONTIËRE DANS LA MONARCHIE DE PAMPELUNE}

Plus à l'ouest, dans les terres soumises à la monarchie de Pampelune, en Aragon et en Navarre, les documents latins emploient parfois le mot »limes«, parfois encore le mot »extrematura« pour désigner les terres faisant face au domaine soumis à l'islam, et c'est en 1059 , pour la première fois dans l'ensemble de l'Occident chrétien, que surgit le mot »frontière « dans le testament du roi Ramire ${ }^{\mathrm{er}}$, lorsque celui-ci emploie l'expression »castros de fronteras«. J'ai montré dans plusieurs articles que l'usage du mot était réduit puisqu'on ne le retrouve que neuf fois entre 1059 et 1209 , tantôt au

11 Céline MARTIN, in confinio externis gentibus. La percepción de la frontera en el reino visigodo, dans: Studia Historica, historia antigua 16 (1998), p. 267-280.

12 Michel ZimmermanN, Le concept de »Marca Hispánica« et l'importance de la frontière dans la formation de la Catalogne, dans: Philippe SÉNAC (dir.), La Marche supérieure d'al-Andalus et l'Occident chrétien, Madrid 1991 (Publications de la Casa de Velázquez. Série archéologie, 15), p. 29-47; Henri DOLSET, Frontière et pouvoir en Catalogne médiévale. L'aristocratie dans l'ouest du comté de Barcelone (début du $\mathrm{X}^{\mathrm{e}}-$ milieu du XII ${ }^{\mathrm{e}}$ siècle), thèse inédite, université de Toulouse 2-Le Mirail 2004, t. 1, p. 29. 
singulier (frontera, fronteram, frontaria, frontarie) tantôt au pluriel (fronteras de mauros). Réservé au souverain, le terme se rapporte alors à un front mobile et son usage se déplace à mesure que la reconquête progresse vers le sud. De manière très suggestive, frontera n'est jamais employé pour désigner les limites du royaume aragonais avec la Castille, la Navarre ou les comtés catalans et c'est donc face à l'islam, dans un moment de tension militaire, que naquit en Occident le mot »frontière « ${ }^{13}$.

\section{LA FRONTIÈRE CASTILLANE}

Paradoxalement, l'emploi du terme est plus tardif en Castille et dans l'Estrémadure. La question de la frontière avait été abordée par Jean Gautier Dalché dans un article consacré à la »Chronica Adefonsi Imperatoris«, puis par Jean-Pierre Molénat à l'occasion du huitième centenaire de la bataille d'Alarcos (1195). Selon ces auteurs, il n'existait pas alors de frontière précise courant à travers les plateaux de l'Estrémadure et de la nouvelle Castille. Il s'agissait au XII ${ }^{\mathrm{e}}$ siècle d'un front militaire mouvant, lié aux aléas des combats. La frontière n'était pas matérialisée par des ouvrages fortifiés et on utilisait alors seulement pour désigner les bornes des terres soumises aux chrétiens les mots »fines«, »extremitas« ou »extremum«. Buresi situe à une date assez tardive, 1171 , la première mention du terme, à deux reprises, dans un fuero octroyé par l'archevêque Cerebrun de Tolède et dans une donation du roi Ferdinand II à l'ordre de Santiago. Le terme se rapporte non pas à une ligne fortifiée, mais à une zone instable, liée aux progrès ou au revers des Castillans face aux Almohades ${ }^{14}$.

En résumé, il semble donc que les études consacrées à la frontière du côté chrétien révèlent que celle-ci constituait une réalité à géométrie variable et qu'une évolution

13 Philippe SÉNAC, Frontière et Reconquête dans l'Aragon du XI ${ }^{\mathrm{e}}$ siècle, dans: ID. (dir.), Frontières et espaces pyrénéens au Moyen Âge, Perpignan 1992, p. 47-60; ID. (en collaboration avec André BAZZANA), Frontières, peuplement et >reconquête` dans la péninsule Ibérique au Moyen Âge, dans: Cahiers de Commarque (1995), p. 103-124; ID., Islam et chrétienté dans l'Espagne du haut Moyen Âge: la naissance d'une frontière, dans: Studia Islamica 89 (1999), p. 89-105; ID., La frontière aragonaise aux $\mathrm{XI}^{\mathrm{e}}$ et $\mathrm{XII}^{\mathrm{e}}$ siècles: le mot et la chose, dans: Cahiers de Civilisation médiévale 42 (1999), p. 259-272; ID., »Ad castros de fronteras de mauros qui sunt per facere«, dans: Carlos DE AYALA MARTINEZ, Pascal BuRESI, Philippe Josserand (dir.), Identidad y representación de la frontera en la España medieval (siglos XI-XIV), Madrid 2001 (Collection de la Casa de Velázquez, 75), p. 205-221; ID., Frontières et reconquêtes dans la péninsule Ibérique ( $\mathrm{XI}^{\mathrm{e}}-\mathrm{XII}^{\mathrm{e}}$ siècles), dans: Jean-Christophe ROMER (dir.), Face aux barbares. Marches et confins d'empires de la Grande Muraille au rideau de fer. Cycle de conférences 2001-2002 du Centre d'études d'histoire de la défense, Paris 2004, p. 31-52.

14 Jean GAUTIER-DALCHE, Islam et chrétienté en Espagne au XII ${ }^{\mathrm{e}}$ siècle. Contribution à la notion de frontière, dans: Hespéris-Tamuda XLVI (1959), p. 183-217; Jean-Pierre MolENAT, Les diverses notions de »frontière« dans la région de Castilla la Mancha au temps des Almoravides et des Almohades, dans: Actas del congreso internacional del VIII centenario de la batalla de Alarcos, éd. Ricardo Izquierdo BENITO, Francisco Ruiz GoMEZ, Cuenca 1996 (Estudios/Universidad de Castilla-La Mancha, 37), p. 15-24; Pascal BuRESI, Nommer, penser les frontières en Espagne aux $\mathrm{XI}^{\mathrm{e}}-\mathrm{XIII}$ e siècles, dans: DE AYALA MARTINEZ, BURESI, JosSERAND (dir.), Identidad y representación de la frontera (voir n. 13), p. 51-74. 
commune se dessine: d'abord perçue comme un espace refuge peuplé de marginaux ( $\mathrm{VIII}{ }^{\mathrm{e}}-\mathrm{IX}^{\mathrm{e}}$ siècles), la frontière devient ensuite un espace de concentration démographique $\left(\mathrm{X}^{\mathrm{e}}-\mathrm{XI}^{\mathrm{e}}\right.$ siècles) et enfin un front militaire qui se déplace progressivement et par saccades vers le sud au gré de la reconquista. L'existence de phases de reculs territoriaux ou de poches de résistance en rend la cartographie complexe, et, malgré les efforts de Flocel Sabaté et de Javier Mestre dans leur »Atlas de la reconquista. La frontera peninsular entre los siglos VIII y XV« (1998), il reste impossible de cartographier dans le détail l'évolution des limites entre chrétiens et musulmans dans la Péninsule. La frontière et son nom résument donc bien toute l'histoire de l'Espagne chrétienne, mais la localisation des premières mentions du mot demeure une source d'interrogation: au regard des progrès territoriaux enregistrés par les Castillans (prise de Tolède en 1085) et de la précocité des combats contre les musulmans en terre catalane (mort du comte Guifré de Barcelone en 897), on comprend mal pourquoi le terme "frontera«, dans le sens de »front" faisant face aux musulmans, apparut d'abord en Aragon au milieu du XI ${ }^{\mathrm{e}}$ siècle, puis seulement en Catalogne et en Castille au milieu et à la fin du XII ${ }^{\mathrm{e}}$ siècle.

\section{LES HOMMES DE LA FRONTIÈRE}

Le deuxième thème de recherche ayant fait l'objet d'une attention particulière ces dernières années concerne les sociétés de la frontière. Du côté chrétien, l'étude de ces sociétés n'a guère connu de profonds bouleversements depuis les travaux menés par les historiens anglo-saxons et bien résumés par la belle expression de Elena Lourie reprise par James P. Powers »a society organized for war «15. Pour ces historiens, comme pour les médiévistes espagnols tels que Angel García de Cortazar ou Pascual Martínez Sopeina, toutes les activités se développant sur les confins méridionaux des principautés chrétiennes faisant face au domaine de l'islam, à commencer par le royaume de Castille, étaient liées à la reconquête, avec une attention toute particulière pour les milices, les ordres militaires et le thème du caballero villano. Ces questions ont été reprises d'un colloque organisé à Saragosse en 1992, sous le nom de »Las sociedades de frontera en la España medieval «16, où la plupart des communications ont souligné l'omniprésence de la guerre dans la vie de la frontière; ainsi, dans la région de Teruel, en 1232, à Belmonte, une charte de peuplement oblige les paysans à participer trois fois par an à la guerre en terre islamique. D'autres travaux plus récents, comme

15 Elena LOURIE, A Sociey Organized for War: Medieval Spain, dans: Past and Present 35 (1966), p. 54-76; James F. POWERS, A Society Organized for War: The Iberian Municipal Militias in the Central Middle Ages, 1000-1284, Berkeley 1988. On mentionnera également: Robert BARTLETT, Angus MACKAY (dir.), Medieval Frontier Societies, Oxford 1989.

16 Las sociedades de frontera en la España medieval, Saragosse 1993. Peu de temps après, l'université de Saragosse organisait une nouvelle rencontre centrée sur les bouleversements territoriaux et sociaux survenus en terre de frontière: Juan Fernando UTRILla, Carlos LALIENA (dir.), De Toledo a Huesca. Sociedades medievales en transición a finales del siglo XI (1080-1100), Saragosse 1998. 
ceux de Carlos Laliena Corbera concernant l'Aragon, ont révélé que la frontière avait été $\mathrm{du} \mathrm{XI}^{\mathrm{e}}$ au XIII ${ }^{\mathrm{e}}$ siècle, le lieu d'une convergence d'intérêts: le roi y voyait un moyen d'affirmer sa légitimité tout en détournant vers l'infidèle les revendications féodales; les seniores y trouvaient un moyen de conquérir de nouvelles terres et des richesses; le clergé y discernait aussi un moyen d'obtenir des biens et d'affirmer sa place en développant l'idée de guerre sainte; et, par le biais de chartes de franchises, de la Catalogne au Portugal, la paysannerie y trouve un moyen d'affirmer sa liberté juridique à une époque où, dans les vieilles terres du nord se développe le servage ${ }^{17}$.

Du côté musulman, dans le sillage d'un bel article de Pedro Chalmeta consacré au concept de »țagr « - dans lequel l'auteur souligne la spécificité des zones de frontière, tant d'un point de vue fiscal que militaire, culturel et social18 -, de nombreuses enquêtes se sont développées pour souligner le caractère distinct de ces sociétés de frontière, et l'accent a récemment été mis sur trois aspects.

\section{UNE FRONTIÈRE OUVERTE AUX ÉCHANGES}

Le premier réside dans le fait que ces sociétés de frontière ne formaient pas des mondes clos et qu'elles entretenaient de nombreux contacts avec l'ennemi, au-delà de tout clivage politique ou religieux. Sans doute ne faut-il pas accentuer immodérément ces relations dans la mesure où elles furent parfois dictées par des motifs diplomatiques ou militaires et que les emprunts à l'autre s'opérèrent souvent par des voies détournées. Ainsi, dans le domaine linguistique, les nombreux emprunts à l'arabe que l'on voit figurer dès le $\mathrm{x}^{\mathrm{e}}$ siècle dans les sources navarraises ou castillanes, tels que amirate, aldea, aliala, almud, alfitna, almunia, alferez, alfethna, furent la plupart du temps transmis par des populations mozarabes réfugiées en terre chrétienne. Il n'empêche que de nombreux liens existaient de part et d'autre de la frontière et que celle-ci ne constituait pas une barrière imperméable. Dans plusieurs articles et dans un livre consacré aux »Carolingiens et al-Andalus ( $\mathrm{VIII}^{\mathrm{e}}-\mathrm{IX}^{\mathrm{e}}$ siècles) $\ll, \mathrm{j}$ 'ai essayé de montrer qu'au-delà des relations passées entre États, des liens fréquents unissaient les grandes familles des confins, et c'est probablement dans la Marche supérieure que les indices de ces contacts sont les plus nombreux.

Ils furent parfois engagés à l'initiative de chefs arabes révoltés contre Cordoue, comme Sulaymān b. al-A rābī qui se rendit en 777 en Saxe pour solliciter l'appui du roi Charles, mais surtout de chefs muwallades désireux d'acquérir leur autonomie. Ainsi, à l'extrême fin du VIII ${ }^{\mathrm{e}}$ siècle, en 798 , un certain Bahlūl b. Marzūq se rendit à Toulouse pour préparer une offensive franque dans la vallée de l'Èbre, et, quelques années plus tard, un autre chef muwallade, 'Amrūs, proposa une alliance à Charlemagne

17 Carlos Laliena, La formación del Estado feudal. Aragón y Navarra en la época de Pedro I, Huesca 1996 (Colección de estudios altoaragoneses, 42).

18 Pedro Chalmeta, El concepto de thaghr, dans: Philippe SÉnAC (dir.), La Marche supérieure d'al-Andalus et l'Occident chrétien (voir n. 12), p. 15-27. 
contre l'émir omeyyade de Cordoue ${ }^{19}$. Dans un bref passage du »kitāb tarșī al-ahbār«", le chroniqueur al-'Udrī rapporte également que le chef muwallade Furtūn b. Muhammad s'allia avec le roi de Pampelune Sancho Garcés $I^{\text {er }}$ et qu'il combattit à ses côtés contre l'émir 'Abd ar-Raḥmān III pendant la campagne de Mitonia, en $918^{20}$. Vers la même époque, son frère 'Amrūs b. Muhammad s'alliait avec Sancho Garcés $\mathrm{I}^{\mathrm{er}}$ et le comte Bernard de Pallars, pour attaquer d'autres musulmans ${ }^{21}$. Des sources telles que le »Codex Rotense«, composé à la fin du $\mathrm{x}^{\mathrm{e}}$ siècle dans la Rioja révèlent surtout que de fréquentes alliances matrimoniales unissaient musulmans et chrétiens au cours des $\mathrm{IX}^{\mathrm{e}}$ et $\mathrm{X}^{\mathrm{e}}$ siècles: Iñiga, la fille du roi de Pampelune Fortún Garcés épousa ainsi l'émir 'Abd Allāh et quelques années plus tard le gouverneur Muhammad aț-Ṭawīl prenait pour femme Domna Sanzia, la fille du comte aragonais Aznar Galindez II22 qui lui donna quatre fils et une fille, Domna Velazquita ${ }^{23}$. Il serait facile de multiplier les exemples de contacts entre chrétiens et musulmans et on se limitera à rappeler avec Picard que c'est avec l'appui de comtes chrétiens que le hă ğ ì $b$ al-Manșūr attaqua SaintJacques de Compostelle en $996^{24}$. Loin d'être un point de détail, cette perméabilité de la frontière conduirait même à considérer que le clivage confessionnel n'est peut-être pas la seule clé de lecture de l'histoire de l'Espagne.

\section{UN ESPACE DOMINÉ PAR DE GRANDS LIGNAGES REBELLES}

Un autre pôle d'intérêt récent concernant ces sociétés de frontière concerne le rôle grandissant tenus par des grands lignages frontaliers, d'origine arabe, berbère ou muwallade. Ce phénomène est particulièrement sensible dans la Marche supérieure ou, selon la "ğamhara" de Ibn Hazm, chacune des grandes familles musulmanes, arabes (Banū Tuọ̆īb) ou muwallades (Banū Qāsī, Banū Šabrīṭ), firent l'objet d'une histoire individuelle et d'un récit collectif malheureusement perdu, "tawārīhn at-țag̉r« (Les

19 Sur ces alliances: Philippe SÉNAC, Chrétiens et musulmans dans les Pyrénées à la fin du $\mathrm{VIII}^{\mathrm{e}}$ siècle, dans: Tolérance et solidarités dans les pays pyrénéens: actes du colloque tenu à Foix les 18-19-20 septembre 1998, colloque organisé par le conseil général de l'Ariège, les Archives départementales, avec la collaboration de l'université de Toulouse 2-Le Mirail, groupe d'histoire des Pyrénées et al., Saint-Girons 2000, p. 99-109, et ID., Les Carolingiens et al-Andalus $\left(\mathrm{VIII}^{\mathrm{e}}-\mathrm{IX}^{\mathrm{e}}\right.$ siècles), Paris 2002.

20 Al-'Ud drīi, kitāb tarșị̂' al-aḩbār, éd. 'Abd al-'Azīz al-Ahwānī, Madrid 1965, p. 68 (p. 79 de la traduction espagnole).

21 Ibid., p. 42 (p. 43 de la traduction espagnole).

22 José María LACARRA, Textos navarros del Codice de Roda, dans: Estudios de Edad Madia de la Corona de Aragón 1 (1945), p. 243: »Asnari Galindones accepit uxor domna Onneca, Garsie Enneconi regis filia, et genuit Galindo Asnari et Garsea Asnari, et domna Sanzia qui fuit uxor regis Atoele mauro«.

23 Ibid., p. 244: »Rege Atoele genuit de domna Sanzia Abdelmelik, et Ambroz, et Furtunio, et Muza, et domna Belazquita«. La suite des événements révèle qu'il eut encore d'autres fils nommés Yahyā et Lubb.

24 Christophe PICARD, Quelques aspects des relations entre chrétiens et musulmans dans les zones de confins du nord-ouest de la péninsule Ibérique ( $\mathrm{IX}^{\mathrm{e}}-\mathrm{X}^{\mathrm{e}}$ siècles), dans: Cahiers de Saint Étienne (1990), p. 5-26. 
annales de la Marche), évoqué à plusieurs reprises par le chroniqueur al-'Udrī. Le cas le plus remarquable est sans doute celui des Banū Qāsī̄25. Comme on le sait, il s'agissait d'un puissant lignage muwallade originaire de Navarre méridionale et dont l'aire d'influence fut extrêmement vaste puisqu'elle couvrait la plus grande partie du nord d'al-Andalus. Le pouvoir de cette famille était tel qu'au milieu du IX ${ }^{\mathrm{e}}$ siècle, selon la »Chronique d'Alphonse III«, l'un d'eux, nommé Mūsā b. Mūsā, rivalisait avec les autres souverains de la Péninsule et se faisait même appeler par ses sujets »le troisième roi d'Espagne «26. Parmi les autres familles rebelles figurent les Banū Halaf, les Banū Šabrīt et surtout les Banū Tuğīib. En ce sens, du fait de leur éloignement de Cordoue et de la proximité des principautés ennemies avec lesquelles il était possible de conclure des alliances, les marches furent presque continuellement des espaces marqués par des soulèvements visant à affirmer l'autonomie de ces grandes familles. Après avoir tenté de les soumettre, le calife dut changer de politique à la suite de la défaite de Simancas (939) en déléguant une partie de ses attributions aux grands lignages des confins d'al-Andalus, tout en leur accordant le titre de gouverneur de manière héréditaire. Les termes employés par le chroniqueur Ibn Hayyān sont particulièrement éloquents à cet égard:

an-Nāṣir était déçu par l'échec de cette expédition, sans aucune comparaison avec ce qui s'était produit jusque-là et, malheureux de son sort, il avait des pensées sombres et n'était pas juste avec lui-même. On lui conseilla de se distraire et de se dédier à son plus grand plaisir, la construction. On dit qu'il se voua à celle-ci de manière résolue, en fondant az-Zahrā en dessous de Cordoue, mettant dans la beauté et la majesté de ces édifices le repos de son esprit. Il décida aussi depuis cette date de ne plus combattre en personne, déléguant ses pouvoirs à ses valeureux, habiles et résolus $q \bar{a} i d$-s qu'il envoyait chaque année en expédition, se limitant à nommer des gouverneurs pour les villes de la Marche supérieure dans la noblesse locale, les valeureux et tenaces Banū Tuğ̄ìb, les Banū D̄î̀ 'n-Nūn, les Banū Zarwāl, les Banū Ġazlūn, les Banū aț-Ṭawīl, les Banū Razīn et autres, maîtres des frontières et de leurs populations. Il divisa entre eux le pays en lots, les renouvela eux et leurs successeurs annuellement en leur donnant de grandes attributions. Il ne cessa de leur donner des cadeaux s'ils venaient le voir, ou des vivres s'ils s'éloignaient... Cependant, et bien que leurs frontières soient défendues et l'ennemi continuellement attaqué, il ne cessa d'envoyer des expéditions (șawāif) qui partaient dans l'été de chaque année depuis la capitale avec des unités de volontaires, jusqu'aux frontières les plus éloignées [trad. PS]27.

25 Alberto CAÑADA, El posible solar originario de los Banu Qasi, dans: Homenaje a don José María Lacarra de Miguel en su jubilación del profesorado, vol. I, Saragosse 1977, p. 33-38; ID., Los Banu Qasi, dans: Principe de Viana 158-159 (1980), p. 5-96; Simón HAYEK, Los Banu Qasi, dans: Boletín de la Asociación Española de Orientalistas 28 (1992), p. 143-157; Julia PAVÓN, Muladíes. Lectura política de una conversión: los Banū Qāsī (714-924), dans: Anaquel de Estudios árabes 17 (2006), p. 189-202.

26 Yves Bonnaz, Chroniques asturiennes (fin $\mathrm{IX}^{\mathrm{e}}$ siècle), Paris 1987, p. 56: „tertium regem in Hispania«.

27 Ibn Ḥayyān, Al-Muqtabas V, éd. Pedro Chalmeta, Federico CorRIEnTe, Muhammad SubH, Madrid, Rabat 1979, p. 437-438; traduction espagnole: Crónica del califa 'Abdarrahmân III anNâsir entre los años 912 y 942 (al-Muqtabis V), par María Jésus VigUERA, Federico CoRRIENTE, Saragosse 1981, p. 328. 
Si le souverain continua de manifester sa présence en envoyant des troupes et des fonctionnaires chargés d'inspecter les défenses ou de participer aux actions guerrières, en contrepartie les gouverneurs devaient demeurer fidèles au souverain, mener des expéditions contre les infidèles et adresser le montant des impôts à Cordoue. La suite du règne de 'Abd ar-Raḥmān III montre que ces hommes se succédèrent dorénavant de père en fils à la tête des principales cités des thiğür.

\section{UN ESPACE PROFONDÉMENT ISLAMISÉ}

Au-delà de cet esprit rebelle, et contrairement à une opinion longtemps répandue selon laquelle les zones de frontière auraient été moins islamisées et moins arabisées que les provinces de l'intérieur de la Péninsule, la toponymie, les événements survenus dans les marches et la précocité avec laquelle ces régions furent soumises au pouvoir musulman montrent qu'elles furent rapidement intégrées au dār al-islām. Les sites étudiés par les archéologues révèlent une profonde islamisation des villes et des campagnes avec des bâtiments édifiés en grand appareil, à la mode cordouane, des maisons villageoises à entrées coudées, ou encore des mosquées, comme sur le site de Las Sillas, à Marcén. Les répertoires bibliographiques connus sous le nom de țabaqāt en fournissent un indice supplémentaire. Ainsi, si on se limite aux recueils d'al-Hušanī (m. 971) et d'al-Faraḍī (m. 1013), le nombre de savants et de juristes installés dans les zones de marche est tout aussi important que dans les secteurs plus méridionaux, théoriquement à l'abri de la menace. Une récente étude consacrée aux fuqahā' de Huesca, en Aragon, révèle d'abord que ces hommes de religion étaient particulièrement nombreux au regard d'autres secteurs de la Marche supérieure - le recueil intitulé »ahbār al-fuqahā' wa 'l-muhadditīn« d'al-Hušanī, en signale 22 à Huesca alors qu'il n'en mentionne que 14 à Tudèle, deux à Tortose et un seul à Lérida ${ }^{28}$. Quelques années plus tard, dans le »tārīh 'ulamā' al-Andalus«, Ibn al-Faraḍī évoque pour sa part 32 fuqahā' à Huesca. À l'égal des autres juristes andalous de ce temps, ces savants disposaient d'une grande culture religieuse. Ils étaient spécialisés dans le $i l m$, dans les réponses aux litiges qui leur étaient soumis (masāîl), et la plupart avaient pratiqué la riḥla en séjournant à Kairouan, en Égypte ou en Iraq29. L'un d'eux, mentionné par al-'Udīì à l'occasion des funérailles du gouverneur Mūsā b. aṭ-Tawīl en 954, le cadi Mūsā b. Hārūn, aurait

28 À titre de comparaison, le même recueil en signale 43 à Tolède, 34 à Saragosse, 14 à Séville et trois à Mérida.

29 Sur ces personnages: Maribel FIERRO, Arabes, beréberes, maladies y mawālī. Algunas reflexiones sobre los datos de los diccionarios biógraficos andalusíes, dans: Manuela MARÍN, Helena de FELIPE (dir.), Estudios onomástico-biográficos de al-Andalus, vol. III, Madrid 1995, p. 41-54; Maria Luisa AvILA, The Search for Knowledge. Andalusi Scholars and their Travels to the Islamic East, in: Medieval Prosopography 28 (2002), p. 125-139. Voir également, à titre de comparaison: Juan VERNET, El valle del Ebro como nexo entre Oriente y Occidente, dans: Boletín de la Real Academia de Buenas Letras de Barcelona XXIII (1950), p. 249-286; et l'étude de Xavier BALlestin, Prosopografía dels fuqahâ' i 'ulamâ' de la zona oriental del tagr al-A'lâ: Balaga, Lârida, Turtûsa, dans: Homenaje al Profesor José María Forneas Besteiro, vol. IV, Madrid 1994, p. 55-119. 
même rédigé une »Histoire de Huesca« malheureusement perdue, et un autre, Hațțāb b. Ismā̄̄il al-Ḡāfiqī (m. 910), fut șăhịb aș-ṣalāt à Saragosse ${ }^{30}$. Le plus renommé fut Ibrāhīm b. 'Ağannas, qui écrivit un résumé de la »mudawwana«, l'œuvre fondamentale du malikisme ${ }^{31}$. Plusieurs notices biographiques montrent également que ces hommes de religion participèrent activement aux combats contre les chrétiens dans le cadre du ǧiha $\bar{a}$, en particulier dans les premières décennies du $\mathrm{x}^{\mathrm{e}}$ siècle. Ainsi, un certain Muhammad b. Šağa participa à la campagne menée par Muhammad aṭ-Ṭawīl dans les environs de Barcelone en 913 et y trouva la mort ${ }^{32}$. Un autre, connu sous le nom de 'Abd Allāh b. Yahyāa, est décrit comme un homme riche qui se préoccupa sans cesse des affaires de la guerre et qui combattit l'ennemi jusqu'à la fin de ses jours (»wa lam yazal mubāširan li 'l-ḥurūb wa kāna muğâhidan al-'aduwwa bi-hā hattā māta«)33. Un dernier, nommé Aḥmad b. Yūsuf b. Mu’addin (m. 921), se vouait au rachat des captifs musulmans en terre ennemie (»min arḍ al-harb«), en suivant la voie de Dieu (»fĩ sabīl Allāh « $)^{34}$, et la notice que lui consacre Ibn al-Faraḍi précise qu'il parvint à faire libérer à lui seul 150 prisonniers ${ }^{35}$. Plus à l'ouest, en Navarre et dans la Rioja, al-Hušanī rapporte qu'un faqīh nommé 'Āmir b. Mūsā était murābiț dans le qașr de Nájera, tout comme Ni'm al-Halaf b. Abī al-Hașīb à Tudèle, à l'époque des Banū Qāsī.

En somme, à l'échelle des $\underline{t} u \dot{g} u \bar{r}$, ce sont de plus en plus les hommes qui y résidaient et les individus qui franchissaient la frontière qui semblent intéresser maintenant les historiens. De ce point de vue, on soulignera qu'un renversement de tendance se produisit aux abords de l'an mil puisque ce furent plus souvent des chrétiens qui migrèrent vers le sud. À côté de grandes figures comme le Cid ou le Portugais Giraldo Sempavor, la documentation fournit en effet de nombreux indices sur ces chrétiens résidant en terre d'islam, tels ce marchand catalan cité dans les Miracles de Sainte Foy de Conques qui vivait près de Balaguer à la fin du $\mathrm{IX}^{\mathrm{e}}$ siècle, ou ce Barcelonais nommé Ramio qui négociait à Cordoue peu avant le raid de 985 , ou encore ce prince navarrais désigné sous le nom de Ramire qui, enrôlé par les musulmans, vint en 1073 incendier la mosquée de Madīnat Ilbīra. Le dossier est immense et c'est dans ce registre qu'il convient de placer l'attention accordée depuis peu aux mercenaires, aux esclaves et aux captifs ainsi que les études consacrées aux mozarabes ${ }^{36}$, aux Hispani, aux Francos, voire aux juifs, hommes de la frontière par excellence...

${ }_{30}$ Muḥammad b. Hārit al-Hušan̄i, ahbār al-fuqahā' wa 'l-muhaddiținn, éd. Maria Luisa AvILA, Luis MoLINA, Madrid 1992, notice 98.

31 Ibn al-Faraḍī, tārīh 'ulamā' al-Andalus, éd. Ibrāhīm al-Abyārī, Le Caire, Beyrouth 1989, p. 37 , notice 9. Son père et ses deux fils, 'Abd ar-Raḥmān et Aḥmad, se consacrèrent aussi à des études juridiques.

32 Muhạmmad b. Hāarit al-Huušan̄i, aḥbār al-fuqahā' (voir n. 30), notice 213.

33 Ibid., notice 300.

34 Ibid., notice 516.

35 Ibn al-Faraḍī, tārīh 'ulamā' al-Andalus (voir n. 31), p. 68, notice 74.

36 Cyrille Aillet, Mayte Penelas, Philippe Roisse, Existe una identidad mozárabe? Historia, lengua y cultura de los cristianos de al-Andalus (siglos IX-XII), Madrid 2008 (Collection de la Casa de Velázquez, 101). 


\section{HABITATS ET PEUPLEMENT EN TERRE DE FRONTIÈRE}

Le troisième thème qui a fait l'objet d'une attention particulière ces dernières années concerne les structures de peuplement et les habitats en terre de frontière. De ce point de vue, il est clair qu'un déséquilibre sensible se manifeste entre les chercheurs travaillant sur l'Espagne chrétienne et ceux qui s'intéressent à al-Andalus dans la mesure où, malgré la part importante accordée au versant chrétien dans les congresos de arqueología medieval española, les recherches liées aux formes de peuplement s'avèrent encore limitées, peut-être parce que les sources écrites, plus abondantes (chartes de peuplement et fueros) invalident parfois des opérations de terrain. Les enquêtes restent dominées par l'étude des fortifications et de grands sites castraux, comme Loarre, et cela dans une perspective essentiellement castellologique. On objectera qu'en fonction du déplacement progressif de la frontière vers le sud, toute intervention archéologique s'inscrit dans une perspective frontalière, mais il est vrai que le lien avec le peuplement environnant demeure limité. Il en est de même du côté musulman où, à l'exception de sites castillans ayant été des lieux de combats, comme Gormaz, Alarcos ou Calatrava, les recherches consacrées aux frontières dites inférieures` et >moyennes` restent encore très limitées ${ }^{37}$. En dehors des travaux menés dans la région de Tolède et en Castille par Manuel Retuerce Velazco et Sergio Martínez Lillo, la seule enquête faisant exception à cette règle est la thèse inédite de Sophie Gilotte, »L'Estrémadure centreorientale ( $\mathrm{VIII}^{\mathrm{e}}-\mathrm{XIII}{ }^{\mathrm{e}}$ siècles): peuplement et formes d'habitat aux marges d'alAndalus «, soutenue à l'université de Paris IV en septembre 200438. La production la plus abondante est celle qui concerne, après le milieu du XIII ${ }^{\mathrm{e}}$ siècle, les fortifications de frontière du royaume nasride, étudiées tout particulièrement par Manuel Acién Almansa et Antonio Malpica Cuello. De la sorte, la plupart des remarques qui vont suivre s'inscrivent dans le cadre de la Marche supérieure, de loin le $\underline{t} a \dot{g} r$ le mieux étudié ces dernières années, en particulier dans le cadre des colloques »Villa« organisés en collaboration avec la Casa de Velázquez ${ }^{39}$.

\section{UNE FORTE DENSITÉ DE PEUPLEMENT}

Contrairement à une idée répandue selon laquelle les confins d'al-Andalus auraient principalement été occupés par des villes chefs-lieux ou des forteresses chargées de

37 Juan Zozaya (dir.), Alarcos '95, El fiel de la balanza, Tolède 1995 (Colección patrimonio historico de Castilla-La Mancha, 15).

38 Sophie GilOTTE, Émergence et déclin de la structure urbaine musulmane en Estrémadure centre-orientale, dans: Patrice CRESSIER (dir.), Castrum VIII. Le château et la ville. Espaces et réseaux, Madrid 2008 (Collection de la Casa de Velázquez, 108; Collection de l'École française de Rome, 105,8; Castrum, 8), p. 71-88.

39 Philippe SÉNAC (dir.), De la Tarraconaise à la Marche supérieure d'al-Andalus ( $\mathrm{IV}^{\mathrm{e}}-\mathrm{XI}^{\mathrm{e}}$ siècle): les habitats ruraux, Toulouse 2006 (Villa, 1); ID. (dir.), Villes et campagnes de Tarraconaise et d'al-Andalus ( $\mathrm{VI}^{\mathrm{e}}-\mathrm{XI}$ siècle): la transition, Toulouse 2007 (Villa, 2); ID. (dir.), Histoire et archéologie des sociétés de la vallée de l'Èbre (VII ${ }^{\mathrm{e}}-\mathrm{XI}^{\mathrm{e}}$ siècle), Toulouse 2010 (Villa, 3). 
défendre les passages par lesquels l'ennemi pouvait pénétrer, les recherches menées dans ces régions ont révélé que la menace n'affectait nullement la densité de peuplement et que l'implantation humaine y était aussi importante que dans les régions plus méridionales. Entre le Río Aragón et le Río Segre, plusieurs centaines d'établissements ont ainsi été reconnus, qu'il s'agisse de villes, de bourgades, de forteresses, de villages, d'almunias ou de tours. À titre d'exemple, plus de 60 établissements musulmans ont ainsi été découverts dans l'ancien district de Huesca, plusieurs dizaines dans celui de Barbițāniya, et les travaux réalisés dans le secteur de Lérida confirment cette observation $^{40}$. L'affaire est loin d'être négligeable puisqu'il est permis de considérer que ce n'est pas un déséquilibre démographique qui fut à l'origine des succès chrétiens. Sans doute cette densité de peuplement semble-t-elle plus faible dans la Rioja, aux confins de la Castille et de la Marche moyenne, mais ce phénomène résulte vraisemblablement de ce que ces régions furent reprises très tôt par la monarchie de Pampelune, dès le début du $\mathrm{X}^{\mathrm{e}}$ siècle, avec la prise de Nájera ou de Viguera sous le règne de Sancho Garcés I ${ }^{\text {er }}(905-925)$.

\section{UN PAYS DE HUSŪN}

La menace pesait cependant sur le peuplement et, conformément à l'expression de Pierre Toubert selon laquelle la frontière est un lieu de »surinvestissement de la puissance publique«, les travaux concernant la frontière supérieure ont également montré qu'il s'agissait d'un espace dominé par des préoccupations défensives: ce phénomène se manifeste d'abord par d'imposantes murailles urbaines, comme à Huesca, Balaguer ou Barbastro, et par un grand nombre de husșun au regard des provinces de l'intérieur. À titre d'exemple, les sources arabes en mentionnent dix dans la région de Huesca, six dans le petit district de Barbastro et surtout 15 autour de Lérida. L'alignement le long des sierras des hușūn les plus septentrionaux de la Marche supérieure suggérerait volontiers l'existence d'une ligne de défense, mais une étude plus détaillée révèle que ces sites, essaimés de la Navarre à la Catalogne, avaient aussi pour fonction de contrôler les sources et l'approvisionnement en eau nécessaire à l'irrigation des terres plus méridionales. Il n'empêche que certaines forteresses de frontière remplissaient une fonction symbolique, comme cette porte d'al-Andalus, que formait le his de Sen et Men, au nord de Huesca, dont la reprise par les musulmans en 942 fit l'objet d'un retentissement particulier et d'une célébration dans la grande mosquée de Cordoue tout

40 Philippe SÉnAC, La frontière et les hommes (voir n. 3); Jiménez J. LORENZO, La dawla de los Banû Qasî. Origen, auge y caída de un linaje muladí en la frontera superior de al-Andalus, Universidad del País Vasco, Vitoria 2008, ou encore celle de Jesús BRUFAL SUCARRAT, L'espai rural del districte musulmá de Lleida (segles XI-XII). Espais de secá meridionals, université de Lérida 2008; Xavier ERITJA I CURIÓ, Entre la Lleida andalusí i la feudal (segles XI-XII). Un model d'explotació rural, dans: Jordi BOLÒS I MASCLANS et al. (dir.), Paisatge i societat a la plana de Lleida a l'Edat Mitjana, Lérida ${ }^{2} 1993$ (Espai/temps, 17), p. 21-44; ID., De l'almúnia a la turris. Organització de l'espai a la regió de Lleida (segles XI i XII), Lérida 1998 (Espai/temps, 35). 
comme dans les quartiers de la capitale ${ }^{41}$. Ces préoccupations défensives se manifestent également par le fait qu'à la différence de l'Andalousie ou du Šarq al-Andalus où prédominent des habitats ouverts ou alquerías gravitant autour d'un his établissements étaient des sites perchés et naturellement défendus. Plus à l'est encore, dans les environs de Fraga et de Lérida, des auteurs comme al-Himyarī ou al-Qazwīn̄ mentionnent enfin des abris souterrains, appelés »sarādīb«, dans lesquels les populations se réfugiaient en cas de menace.

Il y a à Fraga de nombreux abris souterrains qui servent aux habitants à se protéger contre l'ennemi en cas d'attaque de sa part. Chaque abri consiste en un puits d'orifice étroit et qui va en s'élargissant vers le bas. Au fond se trouvent de nombreuses galeries séparées les unes des autres à la manière de celles d'un terrier de gerboise: on ne peut, de la surface du sol, parvenir directement à ces galeries, et l'agresseur n'ose y pénétrer. Si ce dernier enfume le puits d'accès, les réfugiés pénètrent à l'intérieur des galeries et en ferment les portes, pour attendre que la fumée se soit dissipée. En prévision du cas où l'ennemi comblerait le puits d'accès, le souterrain possède une autre communication avec l'extérieur, par laquelle les réfugiés peuvent sortir. Ces souterrains s'appellent chez eux al-fuğ $\bar{u} g$ ' [trad. PS] ${ }^{42}$.

\section{FRONTIÈRE ET ISLAMISATION}

Finalement, le dernier centre d'intérêt pour les archéologues et les historiens du tagigr s'inscrit dans le prolongement de la grande controverse marquée par les travaux de Claudio Sánchez Albornoz sur la repoblación de la Castille au cours des VIII$\mathrm{X}^{\mathrm{e}}$ siècles et concerne le rythme de l'islamisation. Une contradiction apparente se manifeste là entre les données des sources écrites et les enquêtes de terrain qui dépasse largement le cadre des frontières pour rejoindre l'ensemble d'al-Andalus, bien éclairé par les recherches de Sonia Gutiérrez sur des sites tels que le Tolmo de Minateda dans l'arrière-pays du Šarq al-Andalus. Si les récits de la conquête, tout comme les tabaqāt, attestent bien que ces zones de frontière furent soumises très tôt par les musulmans, il semble bien que les secteurs ruraux demeurèrent longtemps à l'écart de l'islamisation. Les recherches archéologiques n'ont fourni aucun mobilier antérieur au milieu du $\mathrm{x}^{\mathrm{e}}$ siècle, $\mathrm{y}$ compris en des sites mentionnés très tôt comme Alquézar, et les recherches menées par Julián Ortega et Carlos Laliena au sud de l'Ėbre confirment ces observations ${ }^{43}$. L'hypothèse la plus probable consiste bien à considérer que l'islamisation, tout comme l'arabisation, s'opéra à la manière d'auréoles concentriques en affectant d'abord les villes, puis un réseau de forteresses nées à l'époque omeyyade, et enfin les campagnes. De la sorte, les fouilles archéologiques réalisées sur le site de Las Sillas, à

41 Philippe SÉNAC, Une forteresse de la Marche supérieure d'al-Andalus, le hisn de Sen et Men, dans: Annales du Midi 181 (1988), p. 17-33.

42 Al-Qazwīnī, Kosmographie, éd. Ferdinand WüstenfelD, Göttingen 1848, p. 429.

43 Carlos LalienA, Julián ORTEGA, Arqueología y poblamiento. La cuenca del río Martín en los siglos V-VIII, Saragosse 2005; Carlos LALIENA, Julián M. OrTEGA, José Antonio BenAVENTE, Los problemas de escala y la escala de los problemas: algunas reflexiones sobre el poblamiento altomedieval en el Bajo Aragón, dans: SÉNAC (dir.), Villes et campagnes (voir n. 39), p. 249262. 
Marcén, me semblent constituer un jalon important puisqu'elles ont révélé que cet établissement associé à une mosquée avait servi vers le milieu du $\mathrm{x}^{\mathrm{e}}$ siècle de point d'attraction pour des populations essaimées jusque-là dans des petits établissements nés de la fragmentation des grands domaines antiques ${ }^{44}$.

À l'issue de cette brève synthèse, il semble donc que l'étude de la frontière connaisse depuis peu de nouveaux développements. Loin d'être un espace spécifiquement lié à la guerre, la frontière est maintenant perçue comme un lieu d'échanges et de contacts, comme un monde entre deux civilisations, dans lequel l'historien de la société et l'archéologue ont trouvé un champ d'étude privilégié. Si la frontière a gagné en épaisseur, et s'il est aujourd'hui difficile d'étudier l'un de ses deux versants sans considérer l'autre, il n'en demeure pas moins qu'au-delà des déséquilibres évoqués au fil de ces pages plusieurs pistes de recherche restent à parcourir. Parmi ces chantiers figure d'abord le lien entre la frontière et l'État, et c'est sans doute ici que le choix de la frontière comme pôle d'observation est apte à éclairer les différences soulignées par Bertrand Badie dans son livre »Les Deux États«. La question rejoint le problème de la territorialité, et si du côté chrétien la thèse de Santiago Palacios intitulée »Fortalezas y poder Político«, a récemment posé la question du lien entre les châteaux de la frontière et la représentation que le souverain castillan avait de son royaume, les toutes dernières thèses consacrées aux taifas de Denia et de Grenade me semblent butter encore sur la question des limites des principautés qui surgirent lors de la fragmentation du domaine omeyyade ${ }^{45}$. Peut-on concevoir un État sans frontière? Cette interrogation rejoint un problème trop rarement abordé, à savoir celui de la cartographie des limites. Du côté chrétien comme du côté musulman, il me semble également que l'on ne s'est pas encore suffisamment intéressé aux frontières intérieures, c'est-à-dire aux clivages à la fois ethniques, confessionnels ou linguistiques en considérant souvent que la frontière séparait en fait deux blocs homogènes. De profondes lignes de partage divisaient chaque versant de la frontière, qu'il s'agisse des mozarabes en terre musulmane ou des mudéjares en terre chrétienne, et de ce fait il est peut-être excessif d'associer systématiquement frontière et reconquête, l'avancée de la seconde ne mettant pas un terme immédiat à la première. Reste la mer, en tant qu'élément de délimitation de l'espace. L'intérêt porté par les Omeyyades au littoral et la présence de ribāt-s le long des côtes conduiraient à penser que la mer était perçue comme une limite politique, mais l'exemple de la taifa de Denia, qui étendit son autorité sur les Baléares et tenta de conquérir la Sardaigne, tout comme celui du califat hammūdide, dont le pouvoir s'étendait de part et d'autre du détroit de Gibraltar autour de Málaga et de Ceuta, compliquent singulièrement l'affaire. L'enjeu est pourtant de taille puisque si l'année 1492 mit fin à une frontière, la même année s'en ouvrit une autre de l'autre côté de l'Atlantique, ce qui conduirait volontiers à considérer, en dernière analyse, que la frontière a horreur du vide...

44 Philippe SÉNAC, Un >village` d'al-Andalus aux alentours de l'an mil. Las Sillas (Marcén, province de Huesca), Toulouse 2009.

45 José Santiago Palacios Ontalva, Fortalezas y poder político. Castillos del reino de Toledo, Guadalajara 2008 (Tierra de Castilla-La Mancha, 8); Travis BRUCE, La taifa de Denia et la Méditerranée $\mathrm{V}^{\mathrm{e}}-\mathrm{XI}^{\mathrm{e}}$ siècle, thèse inédite, université de Toulouse 2-Le Mirail 2009; Bilal SARR, La Granada zirí (1013-1090). Análisis de una taifa andalusí, thèse inédite, université de Grenade 2009. 